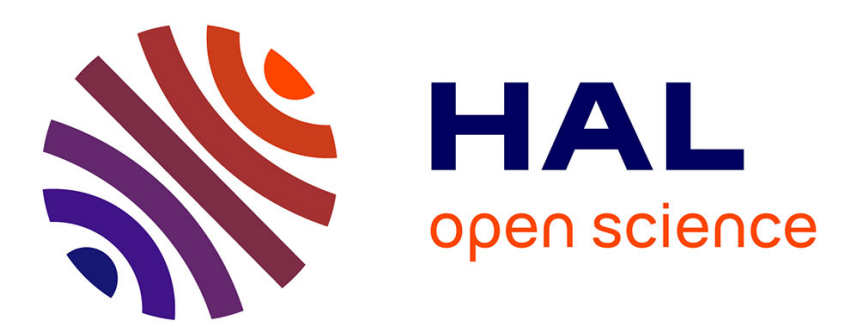

\title{
A decomposition-based optimal control approach for aircraft conflict avoidance performed by velocity regulation
}

Loïc Cellier, Sonia Cafieri, Frédéric Messine

\section{- To cite this version:}

Loïc Cellier, Sonia Cafieri, Frédéric Messine. A decomposition-based optimal control approach for aircraft conflict avoidance performed by velocity regulation. 3rd International Conference on Application and Theory of Automation in Command and Control Systems (ATACCS 2013), May 2013, Naples, Italy. pp.111-113, 10.1145/2494493.2494508 . hal-00909697

\section{HAL Id: hal-00909697 \\ https://hal-enac.archives-ouvertes.fr/hal-00909697}

Submitted on 26 Nov 2013

HAL is a multi-disciplinary open access archive for the deposit and dissemination of scientific research documents, whether they are published or not. The documents may come from teaching and research institutions in France or abroad, or from public or private research centers.
L'archive ouverte pluridisciplinaire HAL, est destinée au dépôt et à la diffusion de documents scientifiques de niveau recherche, publiés ou non, émanant des établissements d'enseignement et de recherche français ou étrangers, des laboratoires publics ou privés. 


\section{A Decomposition-based Optimal Control Approach for Aircraft Conflict Avoidance Performed by Velocity Regulation}

\author{
Loïc Cellier \\ ÉNAC-Univ. of Toulouse \\ F-31055 Toulouse, France \\ loic.cellier@enac.fr
}

\author{
Sonia Cafieri \\ ÉNAC-Univ. of Toulouse \\ F-31055 Toulouse, France \\ sonia.cafieri@enac.fr
}

\author{
Frédéric Messine \\ ÉNSÉÉIHT-Univ. of Toulouse \\ F-31071 Toulouse, France \\ frederic.messine@n7.fr
}

\begin{abstract}
One of the decisive tasks within the air traffic management is the resolution of aircraft conflict avoidance problems. To avoid conflict, aircraft have to preserve a minimal safety distance between them. In this paper, we present optimal control models and approaches based on speed regulation to perform aircraft conflict avoidance. We consider some aircraft configurations with separable trajectories, i.e., such that trajectories of aircraft pairs exhibit conflict zones which are each other separated in terms of time and/or space. We propose a decomposition of the problem in such a way to solve independently subproblems of the original one.
\end{abstract}

\section{Keywords}

Collision avoidance; air traffic management; velocity

regulation; optimal control; decomposition-based approach.

\section{ACM Classification Keywords}

J.2 [Physical Sciences and Engineering]: Aerospace.

\section{INTRODUCTION}

To prevent the risk of collision, the main purpose of Air Traffic Control (ATC) is to ensure a minimum distance of separation between each pair of aircraft. This norm corresponds to $1000 \mathrm{ft}$ vertically and $5 \mathrm{NM}$ horizontally (with the units: $1 \mathrm{NM}$ (nautical mile) $=1,852 \mathrm{~m} ; 1 \mathrm{ft}$ (feet) $=$ $0.3048 \mathrm{~m}$ ). In this context, a pair of aircraft is said in conflict if this norm is not respected.

Different approaches for aircraft Conflict Detection and Resolution (CD\&R) have been proposed (see, e.g., Kuchar and Yang [6] for a survey of existing approaches). Many research works focus on aircraft trajectory deviations, with altitude and/or heading changes. Recently, the European En-Route Air Traffic Soft Management Ultimate System (ERASMUS) project (Bonini et al. [1]) has pointed out the interest in aircraft separation maneuvers based on velocity changes. Thus, new models and solution approaches based on small velocity variations have been developed (see, e.g.,
Pallottino et al. [7], Cafieri [2] ).

We consider optimal control to perform aircraft conflict avoidance based on speed regulation (for an introduction to the optimal control theory, see e.g., Trélat [9]). Starting from our approach combining direct and indirect optimal control methods (Cellier et al. [3]), we propose a decomposition of the problem which exploits the aircraft trajectory topology. This allows us to obtain a more computationally affordable approach for CD\&R.

We recall optimal control for CD\&R based on speed regulation in the next section. We then present the decomposition-based approach. A few remarks and statements of future work conclude the paper.

\section{OPTIMAL CONTROL APPROACH} MODEL WITH ACCELERATION AS COMMAND

We formalize aircraft conflict resolution using optimal control. Taking into account energy criteria, we minimize a quadratic cost penalizing the speed variations, on the set of all the $n$ aircraft $(I)$, during the whole time horizon (from $t_{0}$ to $t_{f}$ ). A dynamic system depending on time $(t)$, allows us from the command - the acceleration variable $(u)-$ to deduce the velocity $(v)$ then, using the trajectory direction $(d)$, the position $(x)$ for each aircraft. Moreover, for operational reasons, the acceleration and velocity are bounded. We consider initial conditions and (free or fixed) terminal conditions on velocities and/or positions. The main constraint is the separation which guarantees, for each pair of aircraft, the fulfillment of the requested norm $(D)$. We start from the following optimal control model, using small speed variations only, and keeping the aircraft trajectories unchanged, to get the aircraft separation.

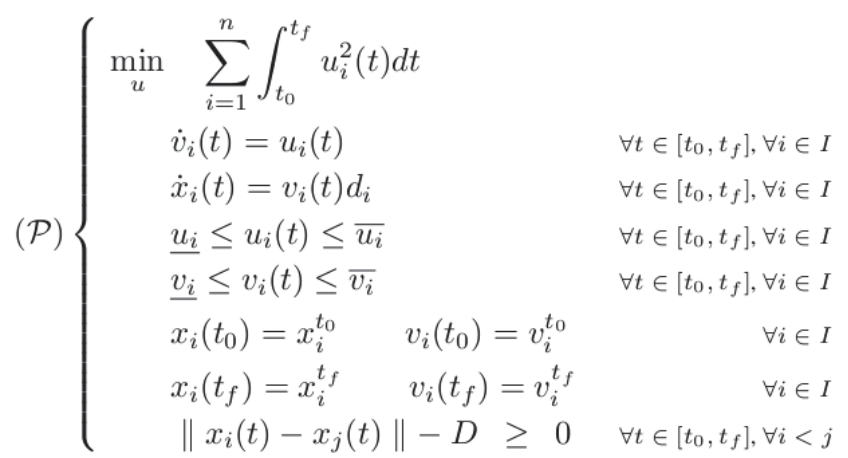




\section{Resolution Strategy}

Optimal Control problems can be numerically solved by using two kinds of methods. First, one can use direct methods. They are generally based on time-discretization and numerical integrators to replace the differential equations. They transform the initial optimal control problem into a large scale NonLinear (continuous) optimization Problem (NLP). A second class of methods is the one of indirect methods, which make it possible to obtain an analytical solution via the Pontryagin Maximum Principle. Nevertheless, for this last one category of numerical optimal control methods, theoretical difficulties exist to manage constraints on the state variables (for more details, see, e.g., Trélat [9]).

In our model, we have to deal with numerous constraints on the state variables. We have constraints on the state variables of first order (velocities), i.e., the velocity bounds, and constraints on the state variables of second order (positions), i.e., the separation conditions. In this context, a direct method appears more easy to implement. It can be applied by performing a time-discretization and replacing the differential equations (representing the system dynamic) by numerical integrators of Euler type. This leads to the resolution of a NLP. In this NLP, the number of variables and constraints largely increases with respect to the number of aircraft involved $(n)$ and the number of considered timesubdivisions $(p)$. More precisely, the computational complexity is given by $O(n p)$ variables (resp. $O\left(n^{2} p\right)$ constraints). In order to reduce the number of variables and constraints, in Cellier et al. [3], we proposed to combine direct and indirect numerical optimal control methods, as briefly recalled below.

We recall the definitions of zone and postzone as follows. Let zone be the region where for an aircraft pair separation constraints have to be verified and postzone be the following region where all the conflicts have been solved and when the aircraft are already separated.

From the spatial point of view, let $x^{\mathrm{ij}}$ enter be the first (by chronological order) trajectory point of the aircraft $i$ for which the distance between this point and another point of the trajectory of aircraft $j$ corresponds to the separation norm. Reciprocally, by projections, we can denote $x^{\mathrm{ij}}{ }_{\text {exit }}$ the last (by chronological order) trajectory point of aircraft $i$ for which the distance between this point and another point of the trajectory of aircraft $j$ corresponds to the separation norm. Similarly, for aircraft $j$, we can define the trajectory points $\left(x^{\mathrm{ji}}{ }_{\text {enter }}\right)\left(\right.$ resp. $x^{\mathrm{ji}}$ exit) to enter (resp. to exit) to the zone.

The same decomposition can be formulated from the point of view of time.

Let $t_{1}^{i \text { min }}$ be the minimum instant time for aircraft $i$ (using upper velocity bound) to reach its first trajectory point $x^{\mathrm{ij}}$ enter (with $j$ another aircraft). Let $t_{2 \_ \text {max }}^{\mathrm{i}}$ be the maximum instant time for aircraft $i$ (using lower velocity bound) to reach its last trajectory point $x^{\mathrm{ij}}{ }_{\text {exit }}$ (with $j$ another aircraft).
Let $t_{1}$ be the zone entry time:

$$
t_{1}:=\min _{i \in\{1 \ldots n\} \backslash\left\{i_{\mathrm{m}_{1}}\right\}} t_{1 \_ \text {min }}^{i} \text {, s.t. } i_{\mathrm{m}_{1}}:=\underset{i \in\{1 \ldots n\}}{\operatorname{argmin}} t_{1 \_ \text {min }}^{i} .
$$

Let $t_{2}$ be the zone exit time:

$$
t_{2}:=\max _{i \in\{1 \ldots n\} \backslash\left\{i_{\mathrm{m}_{2}}\right\}} t_{2 \_ \text {max }}^{i} \text {, s.t. } i_{\mathrm{m}_{2}}:=\underset{i \in\{1 \ldots n\}}{\operatorname{argmax}} t_{2 \_ \text {max }}^{i} .
$$

The zone (from $t_{1}$ to $t_{2}$ ) corresponds to an unique period for 11 the $n$ aircraft. We limit ourselves to satisfy the separation constraint within the zone to guarantee the separation overall.

From $t_{0}$ to $t_{2}$, we apply a direct method. From $t_{2}$ to $t_{\mathrm{f}}$, we do not need to impose the original state variable constraints (velocity bounds and separation constraints). We apply an indirect optimal control method, which gives an analytical solution via the Pontryagin Maximum Principle (Pontryagin et al. [8]).

\section{DECOMPOSING THE PROBLEM EXPLOITING AIRCRAFT TRAJECTORY TOPOLOGY}

We develop the optimal control approach described in the previous section, proposing, for certain aircraft trajectory configurations, a problem-decomposition into small subproblems which can be solved independently.

As a basic assumption, we consider aircraft flying along linear trajectories at the same altitude-level, and focus on tactical phases (i.e., short-term potential conflict flight phases). For the present study, we consider in particular air traffic configurations with separable trajectories, i.e., such that trajectories of aircraft pairs exhibit conflict zones which are each other separated in terms of time and/or space. In practice, aircraft defining a conflict zone do not have any interaction with aircraft defining a different zone.

In this way, we can exploit the topology of the aircraft trajectory configurations to decompose the problem and solve it independently on subproblems, considering zone and postzone, and correspondingly direct and indirect numerical optimal control methods, on each of such subproblems.

We decompose the initial set of aircraft into subsets of aircraft as follows. For a pair of aircraft, we can define a potential concourse if a conflict may appear between them as a consequence of a speed change. We consider aircraft subsets such that if an aircraft has a potential concourse with another, the two aircraft are belonging to the same subset. We refer to such a subset as a cluster.

For each cluster of aircraft $(c)$, we can define its own time range $\left[t_{1}^{\mathrm{c}}, t_{2}^{\mathrm{c}}\right]$ corresponding to its zone (defined in the previous section for a set of aircraft). Assuming all the zones separated from each other, we can solve independently all the aircraft subproblems using their respective zone only. This reduces significantly the computational effort to solve the whole problem. 
Computational experiments are carried out using the AMPL [5] environment and the interior point method solver IpOpt [10] for large-scale nonlinear problems. We consider air traffic configuration involving aircraft flying along their straight paths and potential conflicts. The horizontally separation norm is 5NM. Acceleration are bounded, based on Eurocontrol's Base of Aircraft Data (BADA) [4],namelly $u_{i}{ }^{u b}=-u_{i}^{l b}=4000 N M / h^{2}$. Velocities are bounded, based on the ERASMUS project, by a small speed range:

$\left[v_{\mathrm{i}}^{t 0}-6 \% v_{\mathrm{i}}^{t 0}, v_{\mathrm{i}}^{t 0}+3 \% v_{\mathrm{i}}^{t 0}\right]$ (where $v_{\mathrm{i}}{ }^{\mathrm{t} 0}$ is the initial velocity of aircraft $i$ ). Terminal conditions are returning to the initial velocities $\left(v_{\mathrm{i}}{ }^{\mathrm{t} 0}=447 \mathrm{NM} / \mathrm{h}\right)$ at final time $\left(t_{\mathrm{f}}=1 \mathrm{~h}\right)$.

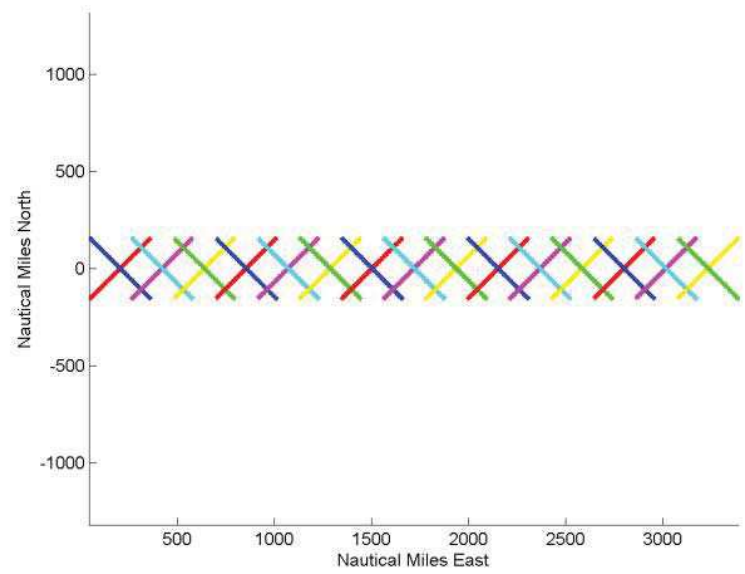

Figure 1. Trajectory configuration involving 30 aircraft and 15 conflicts.

We give an example (see Figure 1) validating our approach. We consider an aircraft configuration involving 30 aircraft and 15 conflicts. The number of pairs of different aircraft corresponds to 435. Applying the previously described strategy based on the zone and the postzone, we are not able to solve the problem. This is due to the large number of variables and constraints in the NLP problem corresponding to the zone. Applying the proposed decomposition approach, based on aircraft clusters of potential concourses, we are able to obtain an efficient solution. The number of constraints is reduced by more than $96 \%$, due to the fact that only 15 pairs of aircraft have to be considered to satisfy the separation conditions. We obtain a (local) optimal solution in 232 seconds (on a laptop with $2.53 \mathrm{GHz}$ and $4 \mathrm{Go}$ RAM), with all conflicts solved (all aircraft separated).

\section{CONCLUSION}

We presented an approach for aircraft conflict avoidance based on optimal control, with an acceleration command, where a decomposition of the problem in such a way to solve independently subproblems of the original one is proposed. Future work will address a further development of this approach to identify subproblems of aircraft involved in more general configurations.

\section{ACKNOWLEDGMENTS}

The authors gratefully acknowledge financial support under grant ANR 12-JS02-009-01 "ATOMIC".

\section{REFERENCES}

1. Bonini, D., Dupré, C., and Granger, G. How ERASMUS can support an increase in capacity in 2020. In Proceedings of CCCT: the 7th International Conference on Computing, Communications and Control Technologies (2009).

2. Cafieri, S. A mixed-integer optimization model for air traffic deconfliction. In Proceedings of GOW: the Global Optimization Workshop (2012).

3. Cellier, L., Cafieri, S., and Messine, F. Hybridizing direct and indirect optimal control approaches for aircraft conflict avoidance. In Proceedings of ADVCOMP: the 6th International Conference on Advanced Engineering Computing and Applications in Sciences (2012).

4. EEC. User manual for the base of aircraft data. Tech. rep., EUROCONTROL Experimental Centre, 2008.

5. Fourer, R., Gay, D., and Kernighan, B. AMPL: a modeling language for mathematical programming Thomson/Brooks/Cole, 2003.

6. Kuchar, J., and Yang, L. A review of conflict detection and resolution modeling methods. IEEE Trans. On Intelligent Transportation Systems 1, 4 (2000), 179-189.

7. Pallottino, L., Feron, E., and Bicchi, A. Conflict resolution problems for air traffic management systems solved with mixed integer programming. IEEE Transactions on Intelligent Transportation Systems 3, 1 (2002), 3-11.

8. Pontryagin, L., Boltyanski, V., Gamkrelidze, R., and Michtchenko, E. Théorie mathématique des processus optimaux. Éditions Mir, Moscou, 1974.

9. Trélat, E. Contrôle optimal : théorie et applications. Vuibert, 2005.

10. Wächter, A., and Biegler, L. On the implementation of primal-dual interior point filter line search algorithm for large-scale nonlinear programming. Mathematical Programming 106, 1 (2006), 25-27. 